\title{
Transmission Through Graphene Junctions with Rashba Spin-Orbit Coupling
}

\author{
M. RATAJ ${ }^{a, b, *}$ AND J. BARNAS ${ }^{a}$ \\ ${ }^{a}$ Faculty of Physics, Adam Mickiewicz University, Umultowska 85, 61-614 Poznań, Poland \\ ${ }^{b}$ The Nano-Bio-Medical Centre, Umultowska 85, 61-614 Poznań, Poland
}

\begin{abstract}
Electronic transport in a graphene junction is considered theoretically. Graphene is assumed to be deposited on a substrate which generates Rashba spin-orbit coupling. However, the Rashba parameters in the two parts of the junction are assumed to be generally different. Additionally, different gate voltages are applied to the two parts, which allow tuning the Fermi level and potential step. We analyze the probabilities of electron transmission through the junction and electrical conductance in the linear response regime.
\end{abstract}

DOI: $10.12693 /$ APhysPolA.127.481

PACS: $72.20 . \mathrm{Fr}, 72.80 . \mathrm{Vp}, 73.23 . \mathrm{Ad}, 73.40 . \mathrm{Lq}, 75.70 . \mathrm{Tj}$

\section{Introduction}

Graphene was the first two-dimensional free-standing crystal available for investigations [1]. After its discovery, one could observe a growing interest in other twodimensional crystals. Nevertheless, graphene is the most thoroughly investigated two-dimensional crystal so far. This is because graphene has outstanding electromagnetic properties, like a long spin coherence length [2] and excellent charge carrier mobility [3], which are promising for future applications [4]. What makes graphene interesting is also its high sensitivity to external perturbations, which sometimes is useful, because it allows adjusting graphene properties to a desired application [5], but sometimes it is undesirable, as it requires extra care to keep stability of a graphene-based device [6]. In this paper we consider two tunable properties: the charge carrier concentration [1] and Rashba spin-orbit coupling [7-9] (RSOC). The former can be tuned by an electrostatic gate potential, while the latter one by a proper atomic or molecular cover layer and a substrate [10-11]. RSOC may be important for future spintronics, mainly because it allows for electrical manipulation of electron spin state [12]. It has been also shown that RSOC can play a crucial role in such phenomena as the spin Hall effect [13], anisotropic magnetoresistance [14], etc. On the other hand, RSOC can lead to undesired spin relaxation in graphene [15].

RSOC appears in graphene due to asymmetry between the cover layer and substrate. An obvious consequence of this is that the coupling should be antisymmetric with respect to the mirror plane which coincides with the graphene plane [16]. In other words, the sign of the Rashba parameter depends on the system configuration. In this paper we consider a graphene junction, in which the Rashba parameters in the two parts of the junction are generally different [17]. We determine transmission across the junction and electrical transport characteristics in the linear response limit.

${ }^{*}$ corresponding author; e-mail: marek.rataj@amu.edu.pl

\section{Model}

To describe charge carriers in pristine graphene we employ the effective continuous model introduced by Wallace [18]

$$
H_{0}=-\mathrm{i} \hbar v_{\mathrm{F}}\left(\sigma_{x} \partial_{x}+\sigma_{y} \partial_{y}\right) \otimes s_{0},
$$

where $v_{F}$ is the electron velocity, $\sigma_{\alpha}$ and $s_{\alpha}$ are the Pauli matrices (for $\alpha=x, y, z$ ) and unit matrices (for $\alpha=0$ ) in the pseudospin and spin spaces, respectively.

Hamiltonian of RSOC as derived by Kane and Mele reads [19]

$$
H_{R}=\lambda\left(\sigma_{x} \otimes s_{y}-\sigma_{y} \otimes s_{x}\right),
$$

where $\lambda$ is the Rashba parameter. The full Hamiltonian of a homogeneous system, $H=H_{0}+H_{R}$, has the following eigenvalues:

$$
E=l \lambda \pm \sqrt{\lambda^{2}+\left(k_{x}^{2}+k_{y}^{2}\right) v_{F}^{2} \hbar^{2}},
$$

where the upper (lower) sign corresponds to the conduction (valence) bands. RSOC leads to band splitting, with $l= \pm 1$ being the corresponding band index. For $\lambda>0$ the conduction (valence) sub-bands with $l=1$ are of higher energy than their counterparts corresponding to $l=-1$. The sub-bands interchange their positions when $\lambda<0$. The sign of $\lambda$ can be changed, for example, by interchanging positions of the cover layer and substrate. Fig. 1 depicts the band structure of graphene for $\lambda<0$ and $\lambda>0$.

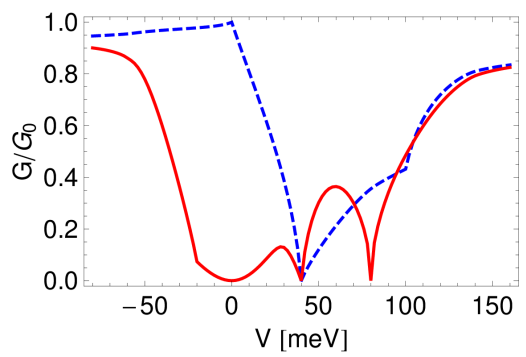

Fig. 1. The band structure of graphene for indicated Rashba parameters. The solid (dashed) lines correspond to the band index $l=1(l=-1)$. The Fermi energy is $E_{\mathrm{F}}=40 \mathrm{meV}$ and in the right panel there is an additional gate potential $V=80 \mathrm{meV}$ which results in the up-shift of the band structure. The other parameters are typical of graphene. 
Assume the two parts of the junction are joined at $x=0$ in the $x y$-plane. We assume the Rashba parameter has different values in the two parts of the junction. We also assume that gate voltages are applied to both parts of the junction, which generate a relative shift of the bands, see Fig. 1, and also allow tuning position of the Fermi level. Thus, Hamiltonian of the junction has the following form:

$$
H=H_{0}+\theta(-x) H_{R, i}+\theta(x) H_{R, t}+\theta(x) V,
$$

where $H_{R, i}$ and $H_{R, t}$ describe RSOC on the incident $(x<$ $0)$ and transmitted $(x>0)$ sides, respectively. In turn $V$ describes the potential step at the junction and the relative shift of the bands.

\section{Electronic transport}

We consider transmission probability from the incident to the transmitted (left to right) side. The eigenvalues in the left $(x<0)$ are given by $E_{i}=l \lambda_{i} \pm$ $\left[\lambda_{i}^{2}+\left(k_{x}^{2}+k_{y}^{2}\right) v_{F}^{2} \hbar^{2}\right]^{1 / 2}$, while in the right part they are $E_{t}=V+l \lambda_{t} \pm\left[\lambda_{t}^{2}+\left(k_{x}^{2}+k_{y}^{2}\right) v_{F}^{2} \hbar^{2}\right]^{1 / 2}$, where $\lambda_{i}$ and $\lambda_{t}$ are the Rashba parameters on the left and right sides. The corresponding eigenfunctions have the form

$$
\psi(\boldsymbol{x})=\frac{1}{N}\left(\begin{array}{c}
l\left(k_{x}-\mathrm{i} k_{y}\right) \\
\mathrm{i} \varepsilon / v_{F} \hbar \\
l \varepsilon / v_{F} \hbar \\
\mathrm{i} k_{x}-k_{y}
\end{array}\right) \mathrm{e}^{\mathrm{i} \boldsymbol{k} \cdot \boldsymbol{x}},
$$

where $N=\sqrt{2}\left[\left|k_{y}\right|^{2}+\left|k_{x}\right|^{2}+\left(\varepsilon / v_{F} \hbar\right)^{2}\right]^{1 / 2}$, while $\varepsilon=E_{i}$ for $x<0$ and $\varepsilon=E_{t}-V$ for $x>0$. In the linear response regime, the relevant transmission corresponds to the Fermi energy, so we assume $E_{i}=E_{t}=E_{\mathrm{F}}$. Let the wave vector component along the junction be $k_{y}$ and the incident wave corresponds to the band index $l$. Since $k_{y}$ is conserved, the scattering problem is effectively one dimensional. The wave function for $x<0$ includes the incident wave as well as the intraband reflected wave (with the reflection amplitude $r_{l, l}$ ) and the interband reflected wave (with the reflection amplitude $r_{-l, l}$ ),

$$
\Psi_{i}(\boldsymbol{x})=\psi_{k_{x}^{(l)}}(\boldsymbol{x})+r_{l, l} \psi_{-k_{x}^{(l)}}(\boldsymbol{x})+r_{-l, l} \psi_{k_{x}^{(-l)}}(\boldsymbol{x}) .
$$

In turn, the wave function on the transmitted side is a combination of the transmitted wave with the conserved index $l$ (with the transmission amplitude $t_{l, l}$ ) and the transmitted wave to the band corresponding to $-l$ (with the transmission amplitude $t_{-l, l}$ ),

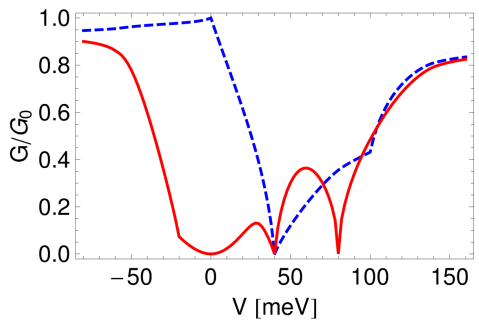

Fig. 2. Conductance of a graphene junction with uniform Rashba coupling $\lambda=30 \mathrm{meV}$ (blue dashed curve), and with opposite Rashba parameters on both sides (red solid line); $\lambda=30 \mathrm{meV}$ on left and $\lambda=-30 \mathrm{meV}$ on the right. In both cases $E_{\mathrm{F}}=40 \mathrm{meV}$.

$$
\Psi_{t}(\boldsymbol{x})=t_{l, l} \psi_{q_{x}^{(l)}}(\boldsymbol{x})+t_{-l, l} \psi_{q_{x}^{(-l)}}(\boldsymbol{x}) .
$$

In the above equations, $k_{x}^{(l)}$ and $k_{x}^{(-l)}$ are the $x$ components of the wave vectors for $x<0$, whereas $q_{x}^{(l)}$ and $q_{x}^{(-l)}$ are the $x$-components of the wave vectors for $x>0$. By utilizing the boundary condition $\Psi_{i}(x=0, y)=\Psi_{t}(x=0, y)$ we find the transmission amplitudes. Taking into account the group velocities one obtains the transmission probabilities $T_{l^{\prime}, l}=$ $\left(v_{t}^{\left(l^{\prime}\right)} / v_{i}^{(l)}\right)\left|t_{l^{\prime}, l}\right|^{2}$. Finally, integration over the incidence angle $\phi$ gives the conductance of the junction, $G=$ $G_{0} \sum_{l} \int d \phi \frac{k_{F}^{(l)}}{k_{F}}\left(T_{l, l}+T_{-l, l}\right) \cos (\phi)$, where $k_{F}^{(l)}$ is the Fermi wave vector of the incident electrons, $k_{F}$ is the average Fermi wave vector of the incident electrons when the Fermi level crosses the two sub-bands on the incidence side and $\phi=\arcsin \left(k_{y} / k_{F}^{(l)}\right)$. The normalization constant is $G_{0}=2 \mathrm{e}^{2} W k_{F} /(h \pi)$, where $e$ is the electron charge, and $W$ is the sample width.

\section{Numerical results}

Consider first the uniform RSOC $\left(\lambda_{t}=\lambda_{i}\right)$ and analyze the conductance dependence on the potential step $V$, see Fig. 2. The Fermi level is assumed to cross the lower conduction subband only $(l=-1)$ on the incidence side. For $V=0$ the system is uniform and the conductance reaches a maximum, $G / G_{0}=1$. When $V$ is growing (decreasing), the band structure in the right part moves upwards (downwards), see Fig. 1. The most characteristic feature is the conductance suppression, $G=0$, at the point where the potential step is equal to the Fermi energy, $V=E_{\mathrm{F}}$. The Fermi level on the right side intersects then the point where the bands touch each other. When $V$ departs from this point, the conductance grows, because the range of angles for which there are states on the right side to which the electrons can be transmitted increases, see Fig. 3 (the plots of $T_{l^{\prime}, l}$ are symmetric with respect to $\phi=0)$. The slope for $V<E_{\mathrm{F}}$ is steeper because the transmission occurs then between the bands with equal index $l$ (intraband transmission) and therefore the wave function matching is better than for $V>E_{\mathrm{F}}$, where only the interband transmission is allowed (compare (a) and (b) in Fig. 3). For $V<0$ the transmission probability of electrons incident at large angles is reduced (see Fig. 3a). Accordingly, the conductance is reduced below its maximum value, and drops gradually with decreasing $V$. Additionally, for $V<E_{\mathrm{F}}-2 \lambda$ the states belonging to the band with opposite $l$ become available for transmission (see Figure 3b), but this transmission is rather small and does not result in any significant increase in the conductance. This is due to relatively poor wave function matching between the states with opposite $l$. In turn, when $V$ grows above $E_{\mathrm{F}}$, the conductance also grows due to the interband transmission, but the wave function matching is poor, so the increase in conductance is rather small. The intraband transmission is possible only when $V>E_{\mathrm{F}}+2 \lambda$, and this results in an abrupt increase in the conductance slope.However, this 


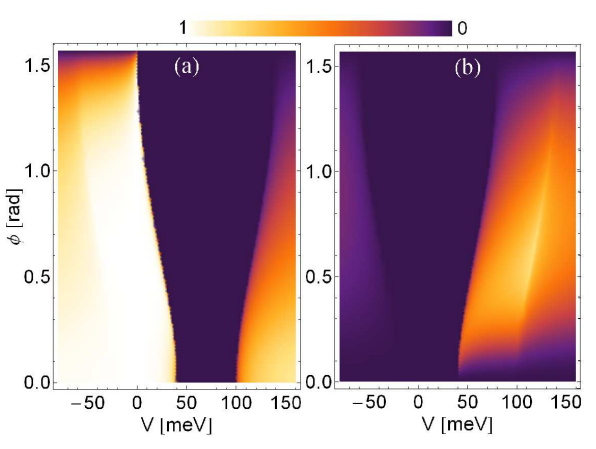

Fig. 3. Transmission probability $T_{-,-}$(a) and $T_{+,-}$(b) through the graphene junction for $\lambda_{i}=\lambda_{t}=30 \mathrm{meV}$ and $E_{\mathrm{F}}=40 \mathrm{meV}$.

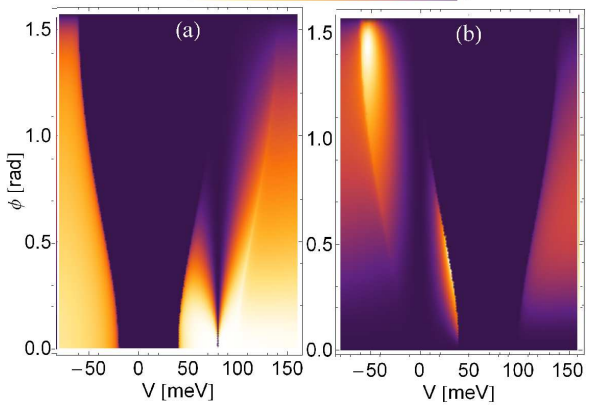

Fig. 4. Transmission probability $T_{+,-}$(a) and $T_{-,-}$(b) for $\lambda_{i}=30 \mathrm{meV}, \lambda_{t}=-30 \mathrm{meV}$ and $E_{\mathrm{F}}=40 \mathrm{meV}$.

slope becomes reduced to zero with a further growth of $V$ ( $G$ saturates).

Let us consider now the conductance for the nonuniform RSOC, $\lambda_{t}=-\lambda_{i}$. From Fig. 2 follows that there are then two additional points where the conductance is suppressed, $V=0$ and $V=2 E_{\mathrm{F}}$. As before, the suppression takes place also for $V=E_{\mathrm{F}}$. However, the slope for $V$ close to $V=E_{\mathrm{F}}$ is now steeper for $V>E_{\mathrm{F}}$ because only intraband transmissions are then allowed. In turn for $V<E_{\mathrm{F}}$ only the interband transmission is possible, which results in a relatively low conductance. When $V$ drops to zero, $V=0$, the transmission is totally suppressed again. In the transmitted part the only available states at the Fermi level are then exact counterparts of the incident states but with opposite $l$, hence they are orthogonal, and all incoming waves are reflected, see Fig. 4b. For $V<0$ the transmission is again nonzero. Moreover, when $V$ drops below $E_{\mathrm{F}}-2\left|\lambda_{t}\right|$, intraband transmission becomes admitted and the conductance increases faster. Let us get back to $V>E_{\mathrm{F}}$ (interband transmission). The rapid initial growth of conductance with increasing $V$ is quickly reduced and changes sign so the conductance becomes totally suppressed at $V=2 E_{\mathrm{F}}$. To find the origin of this suppression one needs to analyze the corresponding expression for the transmission amplitudes. From the boundary conditions follows that the transmission amplitude $t_{l, l}$ is proportional to the factor $\Phi=E_{\mathrm{F}}\left(k_{x}^{(-l)}+q_{x}^{(-l)}\right)-k_{x}^{(-l)} V$, where $\left(k_{x}^{(-l)}\right)\left(\left(q_{x}^{(-l)}\right)\right)$ is the $x$-component of the wave vector corresponding to the wave reflected (transmitted) to the band with opposite band index. Around $V=2 E_{\mathrm{F}}$ both reflection and transmission is possible only to the states with the same band index as the incoming wave, therefore $k_{x}^{(-l)}$ and $q_{x}^{(-l)}$ are both imaginary. Moreover, they are equal for
$V=2 E_{\mathrm{F}}$. Thus, the transmission probability $t_{l, l}$ vanishes for allincidence angles for $V=2 E_{\mathrm{F}}$, which leads to suppression of the conductance, $G=0$, at this point.

In conclusion, we have investigated a specific class of graphene junctions, in which spin-orbit Rashba interaction in the two parts of the junction corresponds to opposite Rashba parameters. Electrical conductance of such junctions is suppressed at three different values of the potential step height $V ; V=0, V=E_{\mathrm{F}}$ and $V=2 E_{\mathrm{F}}$. Physical origins of the conductance suppression at these points are generally different.

\section{Acknowledgments}

The research has been carried out under the project cofinanced by European Union from European Regional Fund within Operational Programme Innovative Economy.

\section{References}

[1] K.S. Novoselov, A.K. Geim, S.V. Morozov, D. Jiang, Y. Zhang, S.V. Dubonos, I.V. Grigorieva, A.A. Firsov, Science 306, 666 (2004).

[2] B. Dlubak, M.-B. Martin, C. Deranlot, B. Servet, S. Xavier, R. Mattana, M. Sprinkle, C. Berger, W.A. De Heer, F. Petroff, A. Anane, P. Seneor, A. Fert, Nature Physics 8, 557 (2012).

[3] S.V. Morozov, K.S. Novoselov, M.I. Katsnelson, F. Schedin, D.C. Elias, J.A. Jaszczak, A.K. Geim, Phys. Rev. Lett. 100, 016602 (2008).

[4] K.S. Novoselov, V.I. Fal'ko, L. Colombo, P.R. Gellert, M.G. Schwab, K. Kim, Nature 490, 192 (2012).

[5] D.W. Boukhvalov, M.I. Katsnelson, J. Phys.: Condens. Matter 21, 344205 (2009).

[6] C. Dean, A.F. Young, I. Meric, C. Lee, L. Wang, S. Sorgenfrei, K. Watanabe, T. Taniguchi, P. Kim, K.L. Shepard, J. Hone, Nature Nanotechnology 5, $722(2010)$.

[7] D. Huertas-Hernando, F. Guinea, A. Brataas, Phys. Rev. B 74, 155426 (2006).

[8] H. Min, J.E. Hill, N.A. Sinitsyn, B.R. Sahu, L. Kleinman, A.H. MacDonald, Phys. Rev. B 74, 165310 (2006).

[9] M. Gmitra, S. Konschuh, C. Ertler, C. AmbroschDraxl, J. Fabian, Phys. Rev. B 80, 235431 (2009).

[10] A. Varykhalov, J. Sánchez-Barriga, A.M. Shikin, C. Biswas, E. Vescovo, A. Rybkin, D. Marchenko, O. Rader, Phys. Rev. Lett. 101, 157601 (2008).

[11] J. Sánchez-Barriga, A. Varykhalov, M.R. Scholz, O. Rader, D. Marchenko, A. Rybkin, A.M. Shikin, E. Vescovo, Diamond Relat. Mater. 19, 734 (2010).

[12] M. Esmaeilzadeh, S. Ahmadi, J. Appl. Phys. 112, 104319 (2012).

[13] A. Dyrdal, V.K. Dugaev, J. Barnaś, Phys. Rev. B 80, 155444 (2009).

[14] M. Rataj, J. Barnaś, Phys. Status Solidi (Rapid Res. Lett.) 7, 997 (2013)

[15] C. Ertler, S. Konschuh, M. Gmitra, J. Fabian, Phys. Rev. B 80, 041405 (2009).

[16] E.I. Rashba, Phys. Rev. B 79, 161409 (2009).

[17] M. Rataj, J. Barnaś, Appl. Phys. Lett. 99, 162107 (2011).

[18] P.R. Wallace, Phys. Rev. 71, 622 (1947).

[19] C.L. Kane, E.J. Mele, Phys. Rev. Lett. 95, 226801 (2005). 